\section{3-Tier Drug Benefit Designs Based on Sound Drug Formulary Principles Will Maximize Favorable Outcomes}

Employer-sponsored drug benefit plans have embraced the 3-tier drug benefit design in dramatic fashion, with $63 \%$ of covered workers subject to a 3-tier copay design in 2003 compared with $27 \%$ just 3 years earlier (in 2000) and 55\% in 2002. ${ }^{1}$ Use of the 2-tier copay design, the predominant drug benefit design in 2000 (with $49 \%$ of covered workers), fell to just $23 \%$ of covered workers in 2003. One major reason for this enthusiasm for 3-tier copay designs is that plan sponsor cost generally declines as member costshare rises. ${ }^{2.4}$ However, when multi-tier copay designs are based upon sound drug formulary principles in which low (favorable) cost-effectiveness ratios determine formulary (tier-2 copay) versus nonformulary (tier-3 copay) status, both beneficiaries and plan sponsors can enjoy lower costs as lower-cost drugs replace the utilization of higher-cost drugs. The use of lovastatin, a tier-1 cholesterol-lowering drug at a cost before copay of approximately $\$ 2$ per day of therapy (with $40 \mathrm{mg}$ ), is about one half the cost for the same outcomes as the tier-3 drug, Mevacor (brand-name equivalent of lovastatin), at about $\$ 4$ per day of therapy at year-end $2003 .{ }^{5}$

In the Kaiser Family Foundation survey of employer-sponsored drug benefit plans, the average member cost-share for tier- 1 drugs such as lovastatin rose by $\$ 2$ in the 3-year period from 2000 to 2003. The average tier- 1 copay was $\$ 7$ in 2000 and $\$ 9$ in 2003, while the cost-share for tier-3 drugs such as Mevacor rose by $\$ 12$, from an average of $\$ 17$ in 2000 to $\$ 29$ in 2003. Clearly, raising tier-3 copays can reduce the proportion of plan-sponsor costs for tier-3 drugs, but how much do these financial incentives and penalties influence drug utilization? The more precise question is, perhaps, how much of a financial incentive is necessary to cause a significant change in the use of preferred versus nonpreferred drugs? A collateral question is how much change in copayments in a drug benefit design is necessary to cause a change in behavior in the use of preferred versus nonpreferred drugs?

There is a growing body of evidence to support our hypothesis that financial incentives in the form of tiered copayments do reduce plan sponsor costs and reduce aggregate drug costs by causing a shift in utilization to generic drugs and lower-cost brand-name drugs. ${ }^{6.7}$ But there is still much to learn about the magnitude of copay differentials necessary to influence a significant proportion of drug plan beneficiaries to change behavior and use preferred (i.e., tier-1 and tier-2 copay) drugs versus nonpreferred (generally nonformulary, tier-3 copay) drugs. In addition to questions surrounding the timing of copayment changes and the magnitude of copayments necessary to influence behavior, other questions that continue to beg for definitive answers include (a) do some patients discontinue useful and valuable drug therapy when faced with tiered copays and (b) what are the clinical and health economic outcomes from discontinued drug therapies, whether these drug therapies are perceived as valuable or of marginal value?

Looking outside prescription drug benefits, data from several studies, including the findings of the Health Insurance Experiment, suggest that (a) copayments reduce the use of medical services, (b) small copayments reduce the use of apparently unnecessary services, and (c) large amounts of cost-sharing reduce the use of medical services such as office visits without selectivity as to the appropriateness of the office visit. ${ }^{8}$ For example, the introduction of a $\$ 5$ copayment for an office visit in the mid-1980s caused an 11\% reduction in visits and $14 \%$ reduction in physical exams, but the $\$ 5$ copayment per office visit had no effect on the immunization of children, screening for cancer in women, or the use of cardiovascular medications. ${ }^{9}$ More than a decade ago, researchers found that, in response to the requirement of a copayment of $\$ 1.50$ for prescriptions, patients selectively reduced the use of discretionary drugs more than their use of drugs deemed essential. ${ }^{10}$ More recently, increases in brand-drug copayments were found to not affect compliance with maintenance medications, ${ }^{11}$ and higher cost-sharing (average 25\%) in employer-sponsored drug plans was associated with higher, not lower, drug utilization compared with lower cost sharing (average 15\%). ${ }^{6}$

In the early 1990s, it was found that a $\$ 10$ increase in copayment, from a copayment of $\$ 25$ to $\$ 35$ per emergency room (ER) visit, resulted in a $14.6 \%$ drop in hospital ER use among a group of 30,276 health maintenance organization members in northern California compared with 2 control groups (60,408 and 37,539 members) without ER copayments. ${ }^{12}$ A separate study of ER patients at 19 hospitals who presented with myocardial infarction (MI) found that an ER copayment requirement ranging from $\$ 25$ to $\$ 100$ did not have an effect on the median length of time from onset of symptoms to arrival at the hospital compared with patients with no ER copayment requirement. ${ }^{13}$

Wogen and Frech in their letter to the editor in this issue of the Journal are correct in their assertion that there is a lack of evidence that higher copayments for prescription drugs result in clinically significant discontinuation rates. ${ }^{14}$ This statement is true despite the recent publication of a study that captured headlines by suggesting that copayments could cause discontinuation of the use of drugs. ${ }^{15}$ In fact, the results of this recent study are mixed and even contradictory. Like many things in life, the truth and wisdom are in the details.

There is a score of shortcomings in the recent study of 3-tier copay designs (for 2 employers), including (a) the significant differences in the age demographics between the intervention and comparison groups, (b) a 2.3-fold difference in the enrollment size between one intervention group and its comparison group, and (c) nondisclosure of the actual dates of implementation of the 3-tier benefit design interventions ("in order to protect the employers' anonymity"). Most important among the findings, however, there were opposite, contradictory effects in discontinuation rates for one of the 3 drug classes that were studied. The rate of discontinuation of angiotensin-converting enzyme (ACE) inhibitors was 16.2\% of tier-3 users in employer 1 versus $6.4 \%$ in its comparison group $(P<0.001)$, but there was an opposite effect for employer 2 in which $8.3 \%$ discontinued use of tier-3 ACE inhibitor drugs versus $15.8 \%$ in the comparison group $(P=0.03)$ This contradictory finding is muddied further by the examination of several alternate therapies, including beta-blockers and calcium channel blockers (as well as histamine-2 receptor antagonists; agents other than statins to lower cholesterol, including cholestyramine, gemfibrozil, or niacin) but 
not examination of the one alternate therapy most likely to explain discontinuation rates of ACE inhibitors, the most logical therapeutic alternative to ACE inhibitors, the angiotensin II receptor blockers (ARBs).

For the other 2 drug classes studied, proton-pump inhibitors (PPIs) and statins, the results of implementation of 3-tier copay designs were inconsistent among the 2 employer groups and their matched comparisons. There was a higher rate of discontinuation of statins for employer 1 versus its comparison $(P=0.04)$ but not for employer 2 and its comparison $(P=0.45)$. There was a higher rate of discontinuation of PPIs for employer 1 versus its comparison $(P<0.001)$ but not for employer 2 versus its comparison $(P=0.79)$. Part of the reason for these apparently disparate findings may lie with the magnitude of the copays and the type of change. Beneficiaries of the employer 1 drug plan were converted from a single-tier copay ( $\$ 7$ for generic or brand-name drug for community pharmacy) to a 3-tier copay design (community pharmacy copayments of $\$ 8$ for generic, $\$ 15$ for preferred brand drugs and $\$ 30$ for nonpreferred brand drugs) compared with a comparison group with an unchanged 2-tier plan of $\$ 8$ copay for generic drugs and $\$ 15$ copay for brand-name drugs. Beneficiaries in employer 2 were confronted with a smaller magnitude and extent of change, transitioned from a 2-tier plan of $\$ 6$ copay for a generic drug and $\$ 12$ for a brand-name drug to a 3-tier plan design with the same copays for tier- 1 and tier- 2 drugs and simply the addition of a third-tier copay of $\$ 24$ for nonpreferred drugs. The comparison group for employer 2 had an unchanged 2-tier plan design of $\$ 6$ and $\$ 12$. Some consumer research suggests that copay differences of as much as $\$ 35$ per prescription are necessary to influence consumer behavior in use of PPIs, ${ }^{16}$ a dollar amount more than twice the tier-3 copayment "penalty" in the 3-tier drug benefit designs in this study of PPIs, ACE inhibitors, and statin drugs.

So, there is much that remains to be answered about the effects of 3-tier drug benefit copay designs on clinical, service-humanistic, and cost outcomes. The important and useful answers will come from carefully designed studies, but even these results will require interpretation since drug formularies upon which 3-tier copay plans are based differ markedly in content from one managed care organization to another. For example, the inconsistent and even contradictory results in the recent study might be explained, in part, by characteristics unique to the study population. The formulary of the pharmacy benefit manager (PBM) contained only 2 statins in tier 3 throughout the study, fluvastatin and lovastatin, and the latter became available by generic name in the middle of the study period. Readers might be better able to judge the importance of this variable if the authors had disclosed the actual patient counts for each of the 3 statin drugs in tier-3, one of which (Mevacor) became available by generic name in December 2000, the midpoint of the study period, and cerivastatin (Baycol), which changed tier-copay status from nonformulary (tier-3 copay) to formulary status (tier-2 copay) in October 2000, also in the midpoint of the study period. Baycol was withdrawn from the market in August 2001, about 5 months prior to the completion of the postintervention study period and 10 months after changing from nonformulary (tier-3 copay) to formulary (tier-2 copay) status for this PBM.

Due, in part, to the content of the tier-3 (nonformulary) statin drugs for this PBM, the utilization of tier-3 statin drugs in this study population was exceptionally low and not generalizable to other patient populations in drug benefit plans. The highest utilization of tier-3 statin drugs was found not in the comparison groups but, ironically, in the intervention (3-tier copay) groups, 3.5\% of 933 patients for employer 2 and $3.4 \%$ of 2,608 statin patients for employer 1 versus $3.1 \%$ of 3,391 statin patients for the comparison group to employer 1 and $1.7 \%$ of the comparison group to employer 2. Therefore, measurement of the effect of the tier-3 plan design change on discontinuation of statin drugs was undertaken on a very small number of patients: 3 (9.1\%) discontinued statin therapy versus 1 patient (4.0\%) for the comparison group $(P=0.45)$ and 19 patients (21.3\%) for employer 1 versus 11 patients (10.6\%) for the comparison group $(P=0.04)$.

Other nuances further complicated the comparisons for the 3 drug classes in this study. In addition to the dual-tier copay status for lovastatin (tier-3 copay [Mevacor] and tier- 1 copay effective December 2000) and the switch in copay for Baycol, followed by its withdrawal from the market, for the PPIs, esomeprazole was a tier-3 PPI before November 2001 but changed to a tier-2 copay in the latter part of the postintervention period, on November 1 , 2001. For the ACE inhibitors, captopril was both a tier- 1 copay drug and a tier-2 (Capoten) copay drug (and most MCOs would place the multiple-source brand drug Capoten in tier-3).

Nevertheless, this recent study of the effects of 3-tier drug copay designs for 2 employers provides a valuable reminder that the answers to the important questions regarding tier-copay drug benefit designs will depend in significant part upon how we ask the questions. It should come as no surprise that neither the answers nor the questions are simple. Behavioral change, including discontinuation rates, will likely depend upon the magnitude of the financial incentive(s) as well as the degree of change from the previous design to the new drug benefit design. When interpreting the results of research conducted on the effects of multi-tier drug benefit plan designs, readers, at a minimum, should assess 2 key variables: (a) the content of the drug formulary tiers and (b) the dollar magnitude of the financial incentives/penalties and the degree of change that are imposed by the new benefit design.

\section{Managed Care Pharmacy- Weighing Clinical, Service, and Cost Outcomes}

The creation of drug benefit plan designs that result in improvement in clinical, service-humanistic, and cost outcomes requires competent and experienced design architects. Managed care pharmacists possess unique knowledge for this task-the combination of information gleaned from clinical trials, experience with assessment of service outcomes, and the ability to identify precisely the price per day of drug therapy. Admittedly, better knowledge of total cost (e.g., direct drug and 